\title{
Human service work, gender and antidepressant use: a nationwide register-based 19-year follow-up of 752 683 women and men
}

\author{
André Buscariolli, ${ }^{1}$ Anne Kouvonen, ${ }^{1,2,3}$ Lauri Kokkinen, ${ }^{4,5}$ Jaana I Halonen, ${ }^{4}$ \\ Aki Koskinen, ${ }^{4}$ Ari Väänänen ${ }^{4,6}$
}

- Additional material is published online only. To view please visit the journal online (http://dx.doi.org/10.1136/ oemed-2017-104803).

${ }^{1}$ Faculty of Social Sciences, University of Helsinki, Helsinki, Finland

${ }^{2}$ SWPS University of Social Sciences and Humanities, Wroclaw, Poland

${ }^{3}$ Administrative Data Research Centre Northern Ireland, Centre for Public Health, Queen's University Belfast, Belfast, UK

${ }^{4}$ Finnish Institute of

Occupational Health, Helsinki, Finland

${ }^{5}$ Faculty of Social Sciences University of Tampere, Tampere Finland

${ }^{6}$ School of Social Policy, Sociology and Social Research, University of Kent, Canterbury, UK

\section{Correspondence to}

Dr Anne Kouvonen, Faculty of Social Sciences, University of Helsinki, Helsinki 00014, Finland;

anne.kouvonen@helsinki.fi

Received 4 October 2017 Revised 22 December 2017 Accepted 9 January 2018 Published Online First 26 January 2018

\section{SLinked}

- http://dx.doi.org/10.1136/ oemed-2018-105017

Check for updates

To cite: Buscariolli $A$, Kouvonen A, Kokkinen L, et al. Occup Environ Med 2018:75:401-406

\section{ABSTRACT}

Objectives To examine antidepressant use among male and female human service professionals.

Methods A random sample of individuals between 25 years and 54 years of age $(n=752683 ; 49.2 \%$ women; mean age 39.5 years). Information about each individual's filled antidepressant prescriptions from 1995 to 2014 was provided by the Social Insurance Institution. First, antidepressant use in five broad human service categories was compared with that in all other occupations grouped together, separately for men and women. Then, each of the 15 human service professions were compared with all other occupations from the same skill/education level (excluding other human services professions). Cox models were applied and the results are presented as HRs for antidepressant use with 95\% Cls.

Results The hazard of antidepressant use was higher among men working in human service versus all other occupations with the same skill/occupational level $(1.22,95 \% \mathrm{Cl} 1.18$ to 1.27$)$, but this was not the case for women $(0.99,95 \% \mathrm{Cl} 0.98$ to 1.01$)$. The risks differed between professions: male health and social care professionals (including medical doctors, nurses, practical nurses and home care assistants), social workers, childcare workers, teachers and psychologists had a higher risk of antidepressant use than men in nonhuman service occupations, whereas customer clerks had a lower risk.

Conclusions Male human service professionals had a higher risk of antidepressant use than men working in non-human service occupations. Gendered sociocultural norms and values related to specific occupations as well as occupational selection may be the cause of the elevated risk.

\section{INTRODUCTION}

Human service professions are characterised by face-to-face interaction in which the employee provides customers with health, educational, social or commercial services. ${ }^{1}$ Because people in these occupations (including medical doctors, nurses, practical nurses, teachers and police officers) often work with particularly vulnerable populations, their performance is linked to experienced emotions. ${ }^{2}$ As such, these professionals engage frequently in emotional labour. They have to suppress their own feelings to 'sustain the outward countenance that produces the proper state of mind in others' ${ }^{3}$ This

\section{Key messages}

What is already known about this subject?

- Human service professionals have high emotional demands at work and they are at a higher risk of antidepressant use.

What are the new findings?

- Hazard of antidepressant use was higher among men working in human service versus men in non-human service occupations in the same skill/occupational level.

- When looking at all human service professionals together, even if the overall prevalence of antidepressant use was higher among women than men, the corresponding risk for these women was not significantly different from the female population working in non-human service occupations.

How might this impact on policy or clinical practice in the foreseeable future?

- The nature of face-to-face interaction, and the emotional labour associated with it, seems to influence mental health outcomes through gender-specific pathways.

can easily create unbalanced relationships which can result in the depletion of one's emotional resources and increase the risk of depressive symptoms. ${ }^{45}$ Indeed, high emotional demands associated with direct client interaction have been linked to affective and stress-related disorders. ${ }^{67}$ However, epidemiological studies on the relationship between emotionally demanding work and the leading mental disorder, depression, have mainly relied on cross-sectional data and self-reported measurements, ${ }^{89}$ which are subject to response bias and the risk of reverse causality.

Emotional demands associated with each occupation have been shown to be gendered. ${ }^{10}$ Related to gendered social roles and labour market structures, women are more often employed in the emotionally demanding human service professions. Tasks involving professional caring and empathising are often typical for female-dominated occupations and less typical for male occupations, ${ }^{11}$ and women are generally more engaged in emotional labour. ${ }^{3} 1213$ Therefore, it can be expected that men working 
in emotionally demanding human service professions may have somewhat different social position and behavioural tendency when compared with their female counterparts. For instance, male nurses have been found to emphasise task-oriented behaviours, instead of people-oriented behaviours, to shape their work role to be more masculine. ${ }^{14}$ The burden of the emotionally taxing occupation may also have adverse gender-specific impacts on mental well-being. In fact, sparse studies on male nurses point to rather poor job satisfaction, ${ }^{15}$ and a tendency to view their job as a 'dead end job'. ${ }^{16}$

To overcome the limitations associated with cross-sectional designs and self-reported measurements, recent studies have used register-based data to investigate mental health in human services professionals. ${ }^{17-19}$ Yet, from the perspective of gendered working life and occupational hazards these studies also have limitations. Their findings are either restricted to specific occupational groups, ${ }^{19}$ or have used short follow-ups. ${ }^{118}$ To fill these gaps, our long-term study uses register data for antidepressant prescriptions for a large representative sample of the Finnish working-age women and men with an exceptionally long follow-up period (1995-2014), and with a comprehensive set of occupational groups. Our hypothesis is that human service professionals have a higher risk of antidepressant use than those working in other occupations with the same skill/education level due to the emotional demands associated with their professions. These impacts might be more pronounced among male than female human service professionals.

\section{METHODS}

\section{Study population}

We obtained a randomly selected 33\% sample of 18-64-year-old permanent residents of Finland in 1994 from the Population Register maintained by Statistics Finland. For this study, we included individuals between 25 years and 54 years of age ( $n=752683 ; 49.2 \%$ women; mean age 39.5 years). This age group was used as the younger professionals would just have entered the profession, whereas those older than 54 years old would retire at some point during the follow-up thereby reducing their exposure time to emotional demands related to their work.

\section{Exposure}

The Finnish Population Register maintained by Statistics Finland includes occupational class for each individual in our data at the end of 1994. The occupations are classified according to the 2001 International Standard Classification of Occupations (ISCO) codes. If the person was not employed at this time, he/ she did not have an occupation and was excluded. To define emotionally demanding work, we identified 21 occupational groups related to human service. The criterion for emotional labour was that the job tasks involved face-to-face interaction in which the employee provides customers with health, educational, social or commercial services. ${ }^{1}$ Professions with small numbers of individuals were grouped together with broader occupational categories based on similar job tasks. For instance, 'Midwifes' was grouped together with the category 'Nurses'. Occupations with low numbers that could not be grouped in any larger occupational categories were excluded (prison guards, firefighters, foreign exchange traders and auditors). The resulting $15 \mathrm{occu}-$ pational groups were further grouped into five larger human service categories following the Wieclaw et al classification: health professionals; education professionals; social workers; customer services workers and miscellaneous (including police officers and psychologists).

\section{Outcome}

Information about each individual's filled antidepressant prescriptions (ie, antidepressant use) (Anatomical Therapeutic Chemical code $\mathrm{N}_{06} \mathrm{~A}^{20}$ ) from 1995 to 2014 was provided by the Social Insurance Institution that maintains the Finnish Prescription Register. This register includes dates of all drug purchases reimbursed to Finnish residents in non-institutional settings. Mean follow-up time until the first antidepressant use was 15.2 (minimum 0.1-maximum 19.0) years.

\section{Covariates}

Information about possible confounders was obtained from the Population Register. For each individual we included sex, age, marital status (married, single, divorced and widowed), income (higher or lower than $€ 17691$ per year), level of education (a university degree vs not), place of residence (Uusimaa vs other counties) and unemployment status (being unemployed for more than 1 month at baseline or not).

\section{Statistical analysis}

Cox proportional hazards regression models were used for data analysis (SAS V.9.4, SAS institute, Cary, North Carolina, USA). Results are presented as HRs for antidepressant use with 95\% CIs. Individuals were followed-up from the 1 January 1995 until the first purchase of antidepressants, death or 31 December 2014, whichever occurred first.

Antidepressant use in the five broad human service categories was first compared with that in all other occupations grouped together. Then each of the 15 human service occupations were compared with all other occupations from the same skill/education level (excluding other human service professions from the reference group). The similarity of skills was determined by the first character of the four-digit ISCO code, which defines broader occupational classes. For instance, the ISCO code for medical doctors is 2221 , the first character ' 2 ' refers to 'specialists', which includes professions like architects, engineers, business specialists, among others. All non-human service professions whose first character was ' 2 ', representing the same skill level, served as the reference group for medical doctors. The code for practical nurses is 5132; other human service professions that also have ' 5 ' as the first character (referring to 'service, sales, and nursing staff') are sellers and retailers, protection and security employees, among others. All non-human service occupations whose first character was ' 5 ' served as the reference group for these professions. This procedure was used to reduce the influence of other work stress factors related to different occupational status groups. The models were adjusted for age, marital status, income, education level, region and baseline unemployment.

\section{RESULTS}

The distribution of human service professionals and the proportions of those with at least one antidepressant purchase during the follow-up by gender are shown in table 1 . Around $11 \%$ of the entire sample $(n=752683)$ were employed in human service professions $(n=85849)$. Among human service professionals, health professionals, education professionals and customer services were the largest groups. Women were more predominantly featured in these professions than men; $85 \%$ of all human service professionals were women. Women had used antidepressants more often than men: in human service professionals, 34\% of women had at least one antidepressant medication purchase during the follow-up compared with $27 \%$ of men. About $19 \%$ 
Table 1 Distribution of human service professionals among male and female employees and the proportions of those with at least one antidepressant purchase during the follow-up ( $n=752683$ )

\begin{tabular}{|c|c|c|c|c|}
\hline & Men & At least one antidepressant purchase & Women & At least one antidepressant purchase \\
\hline & $\mathrm{N}$ & N (\%) & $\mathrm{N}$ & $\mathrm{N}(\%)$ \\
\hline All employees & 382315 & $93211(24)$ & 370368 & 127054 (34) \\
\hline All human service & 12497 & $3343(27)$ & 73352 & 24581 (34) \\
\hline Health professionals & 4175 & $1277(31)$ & 31677 & $10937(35)$ \\
\hline Education professionals & 4106 & $982(24)$ & 22942 & $7226(32)$ \\
\hline Social workers & 933 & $333(36)$ & 4547 & $1741(38)$ \\
\hline Customer services & 1551 & $337(22)$ & 12927 & $4244(32)$ \\
\hline Miscellaneous & 1732 & $414(24)$ & 1259 & $433(34)$ \\
\hline Others & 369818 & 89868 (24) & 297016 & $102473(34)$ \\
\hline
\end{tabular}

of the study participants who had purchased antidepressants had only one purchase during the follow-up while the majority had two or more antidepressant purchases.

Table 2 presents the sociodemographic characteristics of human service professionals and the hazards of antidepressant use by those characteristics. A higher proportion of women had high education compared with men $(52 \%$ and 38\%, respectively). However, men had higher incomes: $85 \%$ of the male population earned more than $€ 17691$ per year, whereas the corresponding figure was only $60 \%$ for women. Men in human service occupations were on average $39.5(\mathrm{SD}=8.3)$ years old, while the mean age among all employed men in our data was $39.5(\mathrm{SD}=8.3)$ years. The proportion of men with a university degree among human service professionals was $62 \%$ and among all employed men $47 \%$.

As table 2 further shows, being divorced and being widowed were associated with an increased hazard of antidepressant use among human service professionals (HR 1.39, 95\% CI 1.36 to 1.41 for divorced men; and HR 1.47, 95\% CI 1.44 to 1.49 for divorced women). Having high income and being unemployed at baseline were associated with an increased use of antidepressants in both genders, whereas living outside of Uusimaa and having a university degree were associated with a slightly decreased hazard. The results of each category were adjusted for other sociodemographic characteristics.

Table 3 presents the hazards of antidepressant use in the five broad categories of human service professions compared with all other occupations from the same skill/education level. The adjusted HR for antidepressant use in all human service professionals together was 1.22 for men (95\% CI 1.18 to 1.27$)$, and 1.00 for women (95\% CI 0.98 to 1.01 ) when compared with non-human service employees. In both women and men, social workers had an increased risk (HR 1.62, 95\% CI 1.46 to 1.81 for men and HR 1.19, 95\% CI 1.13 to 1.24 for women). In education professionals, men had a slightly higher hazard for antidepressant use (HR 1.09, 95\% CI 1.03 to 1.16) when compared with non-human service employees, while the opposite was observed for women (HR 0.92, 95\% CI 0.90 to 0.95 ).

Gender differences could be seen when we look at the 15 specific human service professions separately (see online supplementary table 1 for the unadjusted results, and table 4 for the adjusted results). Elevated hazards for antidepressant use for

Table 2 Sociodemographic characteristics and antidepressant use in human service professionals by gender $(n=85849)$

\begin{tabular}{|c|c|c|c|c|c|c|}
\hline & Men & & & Women & & \\
\hline & $\mathrm{N}$ & $\begin{array}{l}\text { At least one } \\
\text { antidepressant purchase } \mathrm{N}\end{array}$ & $\begin{array}{l}\mathrm{HR}(95 \% \mathrm{Cl}) * \text { for } \\
\text { antidepressant purchase }\end{array}$ & $\mathrm{N}$ & $\begin{array}{l}\text { At least one } \\
\text { antidepressant purchase } \\
\mathrm{N}(\%)\end{array}$ & $\begin{array}{l}\mathrm{HR}(95 \% \mathrm{CI}){ }^{*} \text { for } \\
\text { antidepressant purchase }\end{array}$ \\
\hline \multicolumn{7}{|l|}{ Marital status } \\
\hline Single & 2935 & 753 & 1.00 & 15051 & 4866 & 1.00 \\
\hline Married & 8599 & 2232 & $0.97(0.96$ to 0.98$)$ & 49320 & 15911 & $0.99(0.97$ to 1.00$)$ \\
\hline Divorced & 918 & 346 & $1.39(1.36$ to 1.41$)$ & 7850 & 3399 & 1.47 (1.44 to 1.49$)$ \\
\hline Widow/widower & 45 & 12 & 1.24 (1.12 to 1.37$)$ & 1131 & 405 & 1.21 (1.16 to 1.26$)$ \\
\hline \multicolumn{7}{|l|}{ Education level } \\
\hline No university degree & 7700 & 2035 & 1.00 & 35382 & 11650 & 1.00 \\
\hline University degree & 4797 & 1308 & 0.98 (0.96 to 0.99$)$ & 37970 & 12931 & 0.97 (0.96 to 0.98$)$ \\
\hline \multicolumn{7}{|l|}{ County } \\
\hline Uusimaa & 3513 & 952 & 1.00 & 21513 & 7672 & 1.00 \\
\hline Other counties & 8984 & 2391 & $0.92(0.90$ to 0.93$)$ & 51839 & 16909 & $0.89(0.88$ to 0.90$)$ \\
\hline \multicolumn{7}{|l|}{ Income $(€) \dagger$} \\
\hline$<17691$ & 10565 & 2786 & 1.00 & 43648 & 14409 & 1.00 \\
\hline$\geq 17691$ & 1932 & 557 & $1.22(1.20$ to 1.24$)$ & 29704 & 10172 & 1.08 (1.07 to 1.10$)$ \\
\hline \multicolumn{7}{|c|}{ Unemployment at baseline } \\
\hline$<1$ month & 293620 & 66868 & 1.00 & 292631 & 97357 & 1.00 \\
\hline$\geq 1$ month & 88695 & 23343 & $1.23(1.21$ to 1.25$)$ & 77737 & 29697 & 1.15 (1.14 to 1.17$)$ \\
\hline
\end{tabular}

${ }^{*}$ Adjusted for the other sociodemographic characteristics.

tincome at baseline. 
Table 3 HRs, 95\% Cls for antidepressant use in the five broad human service profession categories, by gender

\begin{tabular}{|c|c|c|c|c|}
\hline & \multicolumn{2}{|l|}{ Men } & \multicolumn{2}{|l|}{ Women } \\
\hline & HR $(95 \% \mathrm{Cl})^{*}$ & HR $(95 \% \mathrm{Cl}) \dagger$ & $\mathrm{HR}(95 \% \mathrm{Cl})^{*}$ & HR $(95 \% \mathrm{Cl}) \dagger$ \\
\hline Non-human serviceł & 1.00 & 1.00 & 1.00 & 1.00 \\
\hline All human service & 1.09 (1.05 to 1.13$)$ & 1.22 (1.18 to 1.26$)$ & 0.95 (0.94 to 0.96$)$ & 1.00 (0.98 to 1.01$)$ \\
\hline Health professionals & 1.28 (1.21 to 1.35$)$ & 1.41 (1.34 to 1.49$)$ & 0.99 (0.97 to 1.01$)$ & 1.04 (1.02 to 1.06$)$ \\
\hline Education professionals & 0.96 (0.90 to 1.02$)$ & 1.09 (1.03 to 1.16$)$ & $0.88(0.86$ to 0.90$)$ & 0.92 (0.90 to 0.95$)$ \\
\hline Social workers & 1.56 (1.40 to 1.74$)$ & $1.62(1.46$ to 1.81$)$ & 1.13 (1.08 to 1.19$)$ & 1.19 (1.13 to 1.24$)$ \\
\hline Customer services & 0.87 (0.78 to 0.97$)$ & 0.95 (0.85 to 1.06$)$ & 0.93 (0.90 to 0.96$)$ & 0.97 (0.94 to 1.00$)$ \\
\hline Miscellaneous & 0.95 (0.86 to 1.05$)$ & $1.12(1.02$ to 1.23$)$ & 0.99 (0.90 to 1.09$)$ & 1.04 (0.95 to 1.15 ) \\
\hline
\end{tabular}

* Unadjusted.

†Adjusted for marital status, education level, county, income and unemployment.

$\ddagger$ All other occupations from the same skill/education level.

both men and women were found for the following professions: medical doctors, practical nurses, home care assistants, childcare workers, social workers, social care workers and special education teachers. In general, however, the associations were stronger in men and the strongest association was found for male social workers (HR 2.11, 95\% CI 1.80 to 2.47). Among primary school teachers, the HR for antidepressant use was 1.15 (95\% CI 1.03 to 1.27 ) in men, and 0.91 (95\% CI 0.85 to 0.97 ) in women. The risk for antidepressant use was also increased among male nurses (HR 1.55, 95\% CI 1.32 to 1.82 ), but not in female nurses, and in male (HR $1.36,95 \%$ CI 1.08 to 1.73 ) but not female psychologists. In addition, women police officers had an increased risk of antidepressant use. On the other hand, male service clerks and women in travel services had a lower hazard of antidepressant use $(0.85,95 \%$ CI 0.75 to 0.98 and $0.83,95 \%$ CI 0.70 to 0.98 , respectively). We also performed sensitivity analyses and modelled the risk of having more than one purchase (a proxy for repeated antidepressant use). All main results remained highly similar, the HR became statistically non-significant only among men working as special education teachers (HR 1.20, 95\% CI 0.94 to 1.53 ) and psychologists (HR $1.27,95 \%$ CI 0.97 to 1.66 ).

Table 4 Adjusted HRs, 95\% Cls of antidepressant use in human service professions when compared with non-human service employees at the same skill/education level, by gender

\begin{tabular}{|c|c|c|c|c|c|c|c|}
\hline \multirow[b]{2}{*}{ Code } & \multirow[b]{2}{*}{ Job title } & \multicolumn{3}{|l|}{ Men } & \multicolumn{3}{|l|}{ Women } \\
\hline & & N/events & $\mathrm{HR}^{*}$ & $95 \% \mathrm{Cl}$ & N/events & $\mathrm{HR}^{*}$ & $95 \% \mathrm{Cl}$ \\
\hline \multicolumn{8}{|c|}{ Health professionals } \\
\hline 2221 & Medical doctors $†$ & $2033 / 587$ & 1.43 & 1.31 to 1.56 & $1967 / 691$ & 1.13 & 1.04 to 1.22 \\
\hline 323 & Nurses $\ddagger \S$ & $509 / 158$ & 1.55 & 1.32 to 1.82 & $12225 / 3998$ & 1.03 & 0.99 to 1.07 \\
\hline 5132 & Practical nurses & $1358 / 449$ & 1.57 & 1.42 to 1.75 & $11881 / 4148$ & 1.17 & 1.13 to 1.22 \\
\hline 5133 & Home care assistants & $275 / 83$ & 1.49 & 1.20 to 1.86 & $5604 / 2100$ & 1.30 & 1.24 to 1.36 \\
\hline \multicolumn{8}{|c|}{ Education professionals } \\
\hline 2321 & Secondary school teacherst & $1955 / 446$ & 1.08 & 0.98 to 1.19 & $4810 / 1468$ & 0.93 & 0.88 to 0.99 \\
\hline 2331 & Primary school teachers $\dagger$ & $1601 / 382$ & 1.15 & 1.03 to 1.27 & $3544 / 1037$ & 0.91 & 0.85 to 0.97 \\
\hline 2332 & Preschool teachers & $125 / 31$ & 1.20 & 0.84 to 1.71 & $2866 / 884$ & 0.98 & 0.91 to 1.05 \\
\hline 2340 & Special education teachers $\dagger$ & $322 / 83$ & 1.23 & 0.99 to 1.52 & $836 / 305$ & 1.14 & 1.02 to 1.28 \\
\hline 5131 & Childcare workersף & $103 / 40$ & 1.72 & 1.25 to 2.36 & $10886 / 3532$ & 1.07 & 1.03 to 1.12 \\
\hline \multicolumn{8}{|c|}{ Social workers } \\
\hline 2446 & Social workerst & $396 / 161$ & 2.11 & 1.80 to 2.47 & $2393 / 929$ & 1.27 & 1.18 to 1.36 \\
\hline 3460 & Social care workers** & $537 / 172$ & 1.57 & 1.35 to 1.83 & $2154 / 812$ & 1.23 & 1.14 to 1.32 \\
\hline \multicolumn{8}{|c|}{ Customer services } \\
\hline 42 & Service clerkst† & $1128 / 241$ & 0.85 & 0.75 to 0.98 & $12246 / 4104$ & 0.99 & 0.95 to 1.03 \\
\hline 511 & Travel services & $423 / 96$ & 1.06 & 0.86 to 1.31 & $481 / 140$ & 0.83 & 0.70 to 0.98 \\
\hline \multicolumn{8}{|c|}{ Miscellaneous } \\
\hline 2445 & Psychologists $\ddagger \ddagger$ & $246 / 69$ & 1.36 & 1.08 to 1.73 & $1131 / 381$ & 1.04 & 0.94 to 1.15 \\
\hline 5162 & Police officers & $1486 / 345$ & 1.05 & 0.93 to 1.19 & $128 / 52$ & 1.40 & 1.06 to 1.84 \\
\hline
\end{tabular}

${ }^{*}$ Adjusted for marital status, education level, county, income and unemployment.

†Medical doctors, secondary school teachers, primary school teachers, preschool teachers, special education teachers, social workers and psychologists were compared with all non-human services occupations whose ISCO code starts with 2 (eg, business, legal and engineering professionals).

‡Includes both '3231: Nurses' and '3232: Midwives'.

$\S N$ urses and social care workers were compared with all non-human services occupations whose ISCO code starts with 3 (eg, finance and sales technicians).

१Practical nurses, home care assistants, childcare workers, travel services and police officers were compared with all non-human services occupations whose ISCO code starts

with 5 (eg, sales persons and demonstrators).

**Includes both '3443: Social benefit administrators' and '3460: Social care workers'.

††Service clerks were compared with all non-human services occupations whose ISCO code starts with 4 (eg, secretaries and insurance clerks).

¥¥Includes both '2412: Counsellors' and '2445: Psychologists'.

ISCO, International Standard Classification of Occupations. 


\section{DISCUSSION}

In this large register-based study, we found that particularly male human service professionals were at a higher risk of antidepressant use than their counterparts within the same occupational grade but working in non-human service occupations. Male health professionals and social workers had the most increased risks. However, it is important to note that the prevalence of psychological distress and depression in the overall population is generally higher among women than men. ${ }^{2122}$ Thus, although the prevalence of antidepressant use among female nurses, and education professionals was higher than that of their male counterparts, the corresponding risk for these employees was not significantly different from the overall female population with a similar skill/educational background.

It has been argued that occupations in which one of the genders is in minority are associated with a higher risk of poor mental health; ${ }^{23}{ }^{24}$ however, this distinction does not account for all the differences found. For instance, although male customer service employees were also a gender minority, they were found to have a lower risk of antidepressant use than their counterparts with similar skill/educational background. One reason for this can be that male customer services employees might have been subjected to work stress factors that are also common to non-human service occupations. What essentially differentiates these occupational groups where gender minorities are common is that health and social care professionals, education professionals and social workers are more likely to be exposed to compassion fatigue, that is, the emphatic exhaustion associated with the care of clients. ${ }^{25}$ Accordingly, the emphatic engagement of health and social care professionals with their patients and clients may make these employees more prone to develop vicarious traumatisation, burn-out syndrome, secondary traumatic stress, compassion fatigue and traumatic counter transference. $^{26-28}$ Thus, our findings suggest the higher risk of antidepressant use in human service professionals is specifically related to the nature of face-to-face interaction and the emotional demands associated with it.

Because personality traits are important determinants of occupational choices $^{29}$ as well susceptibility to depression, ${ }^{30}$ a competing explanation is that men selected into human services are more prone to develop mental illness to begin with, ${ }^{1}$ regardless of the emotional demands associated with their tasks. However, to the best of our knowledge, no previous studies have addressed the relationship between personality traits, occupational choices and susceptibility to depression. Neither had different studies addressing only one of these characteristics observed results that could suggest a converging point. For instance, high levels of openness/intellect are associated with counter gender-stereotypical occupational choices, ${ }^{29}$ whereas high neuroticism, low extraversion and conscientiousness are linked to depression. ${ }^{30}$ Although occupational selection may affect our results, it is not possible to evaluate its magnitude from register-based data.

It is well known that externalising disorders, defined by antisocial behaviour and substance abuse are considerably at a higher level among men than women. ${ }^{30}$ Especially in the masculine occupational cultures (eg, metal industry) behavioural patterns are more inclined towards non-psychological coping strategies. ${ }^{30}$ This may also lead to the underuse of antidepressants among men working in non-human service occupations. Female professional cultures may also explain our findings $(2 ; 15 ; 16 ; 17)$. Male doctors, nurses, teachers and social workers are subjected to a female type of occupational culture, which entails certain behavioural standards and social norms. Among male employees who work in the female dominated sector this may lead to the development of occupation/organisation-specific coping methods (ie, the use of antidepressants) closer to the average of the female rather than the average male population. Men who work in the female dominated professions of caring and empathy may therefore treat themselves in a more feminine way. This may partly explain the high risk of antidepressant use among men working in some human service occupations when compared with other men working in non-human service occupations. However, there may also be occupational selection effects.

\section{Strengths and limitations}

The main strengths of our study include the use of nationally representative register data for antidepressant prescriptions with an exceptionally long follow-up period (1995-2014), and a comprehensive set of occupational groups. The use of self-administered questionnaires is subject to misclassification bias, ${ }^{8}$ but the use of register data reduces this risk since purchase of antidepressant medication is only possible with a clinical diagnosis. Having a comprehensive set of occupational groups allowed us to assess the risk of antidepressant use in different human service professions compared with all other non-human service occupations with the same skill/education level together. This is an important addition to the literature as it allowed us to discuss how the emotional demands of different occupations are associated with antidepressant use. In addition, by including only occupations from the same skill/education level in the reference group we reduced the influence of work stress factors that are not directly associated with emotional demands.

Despite these strengths, our study also had some limitations. First, we used antidepressant use as a proxy for depression, however, even if the main indication of antidepressant use is depression, they are increasingly prescribed to treat other conditions such as anxiety, insomnia and chronic pain. ${ }^{15}$ Second, we cannot infer from the purchase of antidepressants that individuals are effectively adhering to the medication. Whereas our dependent variable was at least one antidepressant purchase during the follow-up, it has been found that up to $50 \%$ of the patients may discontinue the treatment prematurely. ${ }^{31} 32$ Third, the occupation of each individual was determined at the start of the follow-up period and possible changes could not be traced.

\section{CONCLUSIONS}

In this large-scale register study, we found that people working in occupations that contain human service are at increased risk of antidepressant use. The risks, however, differed between occupations and between women and men, the relative risks being the highest among male social workers when compared with the overall male population with the same skill/education level that does not work in human service. Although the incidence of antidepressant use was higher among women in human services professions, it was not significantly different from the overall female population. The nature of face-to-face interaction, and the emotional labour associated with it, seems to influence mental health outcomes through gender-specific pathways. For instance, while male social workers and health professionals had higher risks of antidepressant use, customer service employees had lower risks. In addition, being in a gender minority might also be associated with the observed higher risk of antidepressant use. Finally, the role of gender-specific occupational culture on antidepressant use provides an additional interpretation of our results. 
Contributors $A B, A K o u, L K, J H, A K o$ s and AKPV conceived and designed the experiments, $A B$ and AKos analysed the data, AKos and AKPV contributed to data collection. AKPV is the guarantor of the study. All authors were involved in writing the paper and approved the submitted and published versions.

Funding This study was supported by the Academy of Finland (grant number 267172). AKou was supported by the Economic and Social Research Council (ESRC) (grant number ES/L007509/1) and the Medical Research Council (MRC) (Grant MR/ K023241/1). The funders had no role in the data collection, analysis or writing of the paper.

Disclaimer The corresponding author (AKou) affirms that the manuscript is an honest, accurate and transparent account of the study being reported; that no important aspects of the study have been omitted; and that any discrepancies from the study as planned have been explained.

Competing interests None declared.

Patient consent Detail has been removed from this case description/these case descriptions to ensure anonymity. The editors and reviewers have seen the detailed information available and are satisfied that the information backs up the case the authors are making.

Ethics approval The Ethics Committee of the Finnish Institute of Occupational Health.

Provenance and peer review Not commissioned; externally peer reviewed.

Data sharing statement Because of legal reasons, we are unable to deposit the register data used in the present study with an open data repository. Interested researchers need to obtain these register data directly from the relevant data custodians: the Social Insurance Institution (SII) of Finland and Statistics Finland.

(c) Article author(s) (or their employer(s) unless otherwise stated in the text of the article) 2018. All rights reserved. No commercial use is permitted unless otherwise expressly granted.

\section{REFERENCES}

1 Wieclaw J, Agerbo E, Mortensen PB, et al. Risk of affective and stress related disorders among employees in human service professions. Occup Environ Med 2006;63:314-9.

2 Ogińska-Bulik N. Emotional intelligence in the workplace: exploring its effects on occupational stress and health outcomes in human service workers. Int J Occup Med Environ Health 2005; 18:167-75.

3 Hochschild AR. The managed heart. 2nd ed. California: University of California Press, 2003.

4 Schaufeli WB, Maslach C, Marek T. Professional burnout: recent developments in theory and research. Abingdon, UK: Taylor \& Francis, 2017.

5 Siegrist J. Adverse health effects of high-effort/low-reward conditions. J Occup Health Psychol 1996;1:27-41.

6 Zapf D. Emotion work and psychological well-being. Human Resource Management Review 2002;12:237-68.

7 van VN, de JJ, Söderfeldt M, et al. Quantitative versus emotional demands among Swedish human service employees: moderating effects of job control and social support. Int J Stress Manag 2004;11:21.

8 Tennant C. Work-related stress and depressive disorders. J Psychosom Res 2001;51:697-704.
9 Bonde JP. Psychosocial factors at work and risk of depression: a systematic review of the epidemiological evidence. Occup Environ Med 2008;65:438-45.

10 Acker J. Hierarchies, jobs, bodies. Gender \& Society 1990;4:139-58.

11 Guy ME, Newman MA. Women's jobs, men's jobs: sex segregation and emotional labor. Public Adm Rev 2004;64:289-98.

12 James N. Care = organisation + physical labour + emotional labour. Sociology of Health and IIIness 1992;14:488-509.

13 Morris JA, Feldman DC. The dimensions, antecedents, and consequences of emotional labor. Acad Manag Rev 1996;21:986-1010.

14 Evans J. Men in nursing: issues of gender segregation and hidden advantage. J $A d v$ Nurs 1997;26:226-31.

15 Rajacich D, Kane D, Williston C, et al. If they do call you a nurse, it is always a "male nurse": experiences of men in the nursing profession. Nurs Forum 2013;48:71-80.

16 Walters V, Lenton R, French S, et al. Paid work, unpaid work and social support: a study of the health of male and female nurses. Soc Sci Med 1996;43:1627-36.

17 Madsen IE, Diderichsen F, Burr H, et al. Person-related work and incident use of antidepressants: relations and mediating factors from the Danish work environment cohort study. Scand J Work Environ Health 2010;36:435-44.

18 Thielen K, Nygaard E, Rugulies R, et al. Job stress and the use of antidepressant medicine: a 3.5-year follow-up study among Danish employees. Occup Environ Med 2011;68:205-10.

19 Rantonen O, Alexanderson K, Pentti J, et al. Trends in work disability with mental diagnoses among social workers in Finland and Sweden in 2005-2012. Epidemiol Psychiatr Sci 2017;26:1-11.

20 WHO Collaborating Centre for Drug Statistics Methodology. Guidelines for ATC classification and DDD assignment 2014. Oslo, 2013.

21 Rosenfield S. Sex differences in depression: do women always have higher rates? $J$ Health Soc Behav 1980;21:33-42.

22 Rosenfield S, Vertefuille J, Mcalpine DD, et al. Gender Stratification and Mental Health: An Exploration of Dimensions of the Self. Soc Psychol Q 2000;63:208-23.

23 Kjellberg A. Men, work and health. Women's heal work, 1998:279-307.

24 Evans 0, Steptoe A. The contribution of gender-role orientation, work factors and home stressors to psychological well-being and sickness absence in male- and female-dominated occupational groups. Soc Sci Med 2002;54:481-92.

25 Adams RE, Boscarino JA, Figley CR. Compassion fatigue and psychological distress among social workers: a validation study. Am J Orthopsychiatry 2006;76:103-8.

26 Collins S, Long A. Working with the psychological effects of trauma: consequences for mental health-care workers--a literature review. J Psychiatr Ment Health Nurs 2003;10:417-24

27 Laposa JM, Alden LE, Fullerton LM. Work stress and posttraumatic stress disorder in ED nurses/personnel. J Emerg Nurs 2003;29:23-8.

28 Yoon SL, Kim JH, Stress J-R. Job-related stress, emotional labor, and depressive symptoms among Korean nurses. J Nurs Scholarsh 2013;45:169-76.

29 Woods SA, Hampson SE. Predicting adult occupational environments from gender and childhood personality traits. J App/ Psychol 2010;95:1045-57.

30 Turtiainen J, Väänänen A. Men of Steel? The Masculinity of Metal Industry Workers in Finland after World War II. J Soc Hist 2012;46:449-72.

31 Sansone RA, Sansone LA. Antidepressant adherence: are patients taking their medications? Innov Clin Neurosci 2012;9:41-6.

32 Riihimäki K, Vuorilehto M, Isometsä E. Maintenance pharmacotherapy for recurrent major depressive disorder in primary care: A 5-year follow-up study. Eur Psychiatry 2017;41:111-4. 